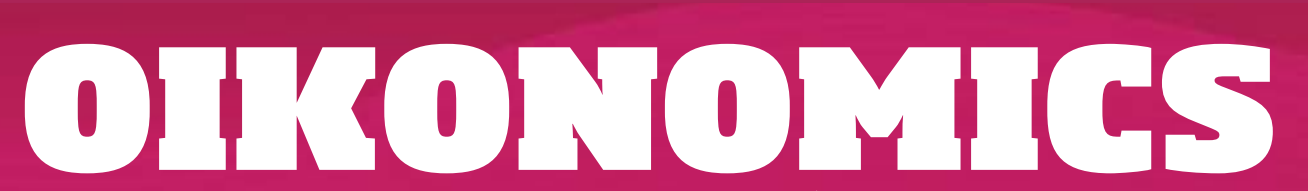

Revista de economía, empresa y sociedad

UN NUEVO ENTORNO

\title{
Las fintech y la reinvención de las finanzas
}

\section{David Igual Molina}

Profesor de la BSM-UPF. Profesor colaborador de la UOC

RESUMEN La industria bancaria se enfrenta a una intensa transformación de su actividad, tanto por la necesidad de reinventar sus servicios (caros y no concebidos para un uso en línea) como por el cambio en las exigencias de productos digitales de los usuarios, así como por la necesidad de ajustar unas estructuras ineficientes. Tradicionalmente, el sector financiero ha sido un ámbito casi exclusivo de las entidades financieras, pero el abaratamiento de las tecnologías ha permitido la aparición de nuevos players en la industria, como son las fintech con propuestas alternativas en todos los ámbitos de la actividad financiera, mediante nuevas fórmulas mobile-first y data-driven. No obstante, después de unos primeros años de vida, la mayoría de estas nuevas empresas presentan problemas de escalabilidad y, a diferencia de su filosofía original, están colaborando con la banca y generan una asociación de mutuo interés: las fintech contribuyen a la transformación del banco, mientras que estas, con el apoyo del banco, alcanzan el crecimiento que por sí solas no conseguían. Mediante estas asociaciones banca-fintech, se da la paradoja de que estas entidades, que inicialmente eran retadoras a los bancos, pueden acabar siendo su punto de apoyo para que el cambio en el sector bancario sea más rápido y más transformacional que disruptivo. Por otra parte, el grupo de fintech que mantiene una competencia con la banca (entre un $20 \%$ y un $25 \%$ ) se ve obligado a movimientos de fusiones, acuerdos, etc. para conseguir llegar al break-even. En España, los problemas de crecimiento parecen aún más intensos que en otros países en estas áreas de competencia con la banca (roboadvisors y crowdlending). 
El problema más importante para la banca proviene de los grandes operadores tecnológicos, que tienen capacidad para desbancar a las entidades financieras en algunos de los ámbitos más rentables de la actividad. Parece imposible de mantener por parte de la banca el total dominio del negocio en las zonas comunes con las tecnológicas, como por ejemplo los pagos de las compras y los envíos de dinero. No obstante, los bancos tienen a su favor el reconocimiento de la gestión de protección de datos de sus clientes, que es un valor en el que superan claramente a las tecnológicas.

Los bancos están desarrollando múltiples estrategias de acuerdos con las fintech, como por ejemplo compras directas, programas de aceleración e incubación, fondos venture capital, acuerdos de servicios y acuerdos de partnership. El análisis correcto de cada área de innovación es determinante para identificar las aportaciones de una fintech, y las variables clave son la capacidad de generar volumen y la de desplazar servicios bancarios actuales. En este artículo, se propone un modelo de relación consistente en una integración gradual de las fintech en los entornos bancarios en forma de: i) integración en el core del banco; ii) acuerdos de colaboración o servicios; iii) contribuir a su desarrollo mediante programas de aceleración e incubación y lanzamiento de concursos o programas challenger para descubrir talento.

PALABRAS CLAVE fintech; ecosistema fintech; digitalización banca; transformación banca; transformación digital finanzas; colaboración bancafintech

\section{FinTech and the reinvention of finance}

ABSTRACT The banking industry is facing a major transformation of its activity due to the need to reinvent its services (which are expensive and not designed for online use), the change in user demand for digital products and the need to adjust inefficient structures. Traditionally the financial sector has been almost exclusively an area for financial institutions, but the falling cost of technologies has led to the emergence of new players in the industry, such as fintechs, with alternative proposals in all spheres of financial activity, through new mobile-first and data-driven formulas. However, after a few years, most of these new companies experience scalability problems and, going against their original philosophy, they end up collaborating with banks, generating a partnership of mutual interest: fintechs contribute to the transformation of the bank, and with the support of the bank, they achieve growth that they would not achieve alone. Through these banking-fintech partnerships, a paradox 
arises in that these entities which initially challenged the banks may end up being their point of support, ensuring the change to the banking sector is quicker and more transformational than disruptive. Conversely, the fintechs that remain in competition with the banks (between 20\% and 25\%) are forced into mergers, agreements, etc. in order to break-even. In Spain, the growth problems in these areas of competition with the banks (robo-advisors and crowdlending) seem even more intense than in other countries.

The most significant problem for banks comes from the large tech operators that have the capacity to unseat financial institutions in some of the most profitable spheres of activity. It seems impossible for banks to maintain total control of the business in the spheres that are shared with tech operators, such as purchase payments and money transfers. However, banks have an advantage in terms of their widely-recognised customer data protection management, which is a value in which they clearly exceed the fintechs.

Banks are developing multiple agreement strategies with fintechs, such as direct purchases, acceleration and incubation programmes, venture capital funds, service agreements and partnership agreements. Proper analysis of each area of innovation is crucial to identify the contributions made by a fintech, and the key variables are the capacity to generate volume and ability to displace current banking services. This article proposes a relationship model consisting of the gradual integration of fintechs into banking environments through: i) integration into the core of the bank; ii) collaboration or service agreements; iii) contributing to their development through acceleration and incubation programmes and the launch of "challenger" programmes or competitions to discover talent.

KEYWORDS fintech; fintech ecosystem; banking digitalisation; banking transformation; digital finance transformation; bank-fintech collaboration

\section{Introducción}

Un microchip insertado en la mano ${ }^{1}$ y capaz de efectuar el proceso de pago en establecimientos comerciales, que elimina no solo las tarjetas de crédito o débito

1. Venmo y otros han efectuado experimentos con éxito mediante la implantación subcutánea de microchips del tamaño de un grano de arroz, que han podido completar el proceso de pago en establecimientos comerciales. 
sino también el pago directo con móvil, un algoritmo que es capaz de efectuar determinados diagnósticos médicos ${ }^{2}$ a partir de un selfie, muy útil en el proceso de contratación de seguros de vida, o la eficacia de los chatbots ${ }^{3}$ para la atención al cliente frente a los típicos call centers, son solo algunos ejemplos de la inmensa cantidad de innovaciones concretas que están apareciendo en el mundo de las finanzas. Muchas de estas aplicaciones, actualmente, solo funcionan en formatos de prototipos y otras están más desarrolladas con implantaciones parciales, pero es evidente que el avance de la tecnología y su abaratamiento para nuevos desarrollos auguran grandes cambios en la actual gestión de las finanzas.

Existe una unanimidad por parte de analistas, expertos, directivos, medios de comunicación, etc. sobre el momento de disrupción en la industria de las finanzas y en particular en el sistema bancario que conocemos, y también existe un acuerdo sobre la rápida velocidad a la que se van a producir los cambios. ${ }^{4}$ De la misma manera que en otros sectores la disrupción ha llevado a la reinvención de industrias como la fotografía (del antiguo concepto proteccionista de Kodak al de la compartición de Instagram), los viajes (de las agencias de viajes físicas a los generadores de ofertas, buscadores o Airbnb) o la publicidad (declive de la eficacia de los anuncios de prensa frente a Google Adwords, Facebook Ads, etc.), las finanzas se transformarán en servicios con prestaciones y formas distintas a las actuales, aún no definidas.

Habitualmente, se hace referencia al impacto de la digitalización y a la aparición de nuevos agentes como los principales elementos generadores de esta transformación, pero los cambios disruptivos son siempre más profundos - no se trata de sustituir oficinas por móvil-, afectan a la esencia misma de la actividad, los

2. MIT TecnologyReview: muchos trastornos genéticos se asocian a características físicas que ayudan a reconocerlos, pero los médicos no los pueden conocer todos. Por eso, el 60 $\%$ de los médicos ya usan aplicaciones como Face2Gene. Existen diferentes aplicaciones más especializadas en diagnósticos oculares o de cáncer de piel a través de algoritmos.

3. Véase «Business Insider: How chatbots and artificial intelligence will save banks and the finance industry billions». En España también tienen aplicaciones, entre otros, CaixaBank con Neo, Santander para sus empleados de la red de oficinas o el BBVA, que cuenta con un asistente de voz para enviar dinero o localizar un cajero cercano.

4. Muchos estudios apuntan a este cambio rápido. Entre otros, se refieren a esta velocidad: PWC, FinancialServicesTechnology 2020 and Beyond: Embracingdisruption; EFMA, WorldRetailBankingReport 2017; Deloitte, Beyond Fintech: A PragmaticAssessmentOfDisruptivePotential In FinancialService; Forrester, Predictions 2018: FinancialServicesCompaniesGetSeriousAbout Digital Transformation; Indra, La nueva banca, una plataforma al servicio de tu bienestar financiero. 
productos anteriores acaban siendo sustituidos por nuevos servicios reinventados, aparecen fórmulas impensables anteriormente. Nuevas compañías desplazan a operadores que han dominado los mercados con los anteriores modelos. En este artículo analizamos el momento actual de la transformación de las finanzas, los principales motores de cambio, la competencia generada por la entrada en los mercados financieros de nuevos players como son las fintech o las grandes compañías tecnológicas (bigtech), y cómo pueden ayudar a reinventar las finanzas.

\section{Motores del cambio}

En primer lugar, hay que destacar las enormes diferencias territoriales existentes en el mundo ${ }^{5}$ en el ámbito de las finanzas, ya que mientras que en los países occidentales el nivel de bancarización es prácticamente total, con unas redes de oficinas muy extendidas, cajeros automáticos omnipresentes, etc., en muchas zonas del mundo - por ejemplo, en África - solo disponen de una cuenta bancaria el 30 \% de la población; y en algunos países latinoamericanos, apenas representan un $60 \%$ los habitantes con servicios bancarios. Es evidente que el desarrollo de las finanzas será distinto en cada zona. Por una parte, en las áreas de mayor bancarización, los nuevos y retadores servicios financieros mobile-first y data-driven pueden provocar una fuerte reestructuración del actual sector bancario. En cambio, se abren muchas opciones de crecimiento en países en los que un servicio financiero mediante oficinas bancarias es inviable, pero mediante canales digitales se puede conseguir una mayor inclusión financiera de sus habitantes.

Además de los cambios en las preferencias de los consumidores, derivados de la digitalización, la banca, después de la reciente crisis, tiene dificultades para alcanzar niveles sostenibles de rentabilidad con el actual modelo de negocio. Por ejemplo, los bancos europeos tienen un RoE (rentabilidad sobre recursos propios) por debajo del coste del capital; ${ }^{6}$ considerando que más del 30 \% de sus costes provienen de las redes de oficinas, el proceso de reestructuración se acelerará y la digitalización será imprescindible, también, del lado de la oferta de productos bancarios.

5. Véase World Bank Group. The Global Findex Database 2017. Measeuring Financial Inclusion and the fintech revolution.

6. Véase EBA report on the impact of fintech on institutions' business models BA (3 de julio del 2018, pág. 9). También Indra, en su informe La nueva plataforma al servicio de tu bienestar financiero (2017, cap. 1). 
Otro motor de aceleración del cambio es el derivado de la profusión de nuevas tecnologías y sus posibilidades reales en la banca, que obligan a diseñar estrategias de implantación y generación de capacidades, a desarrollar casos de uso para luego generalizar, a disponer de recursos humanos adecuados, etc., para no quedar al margen de las grandes ventajas de la innovación. Este cambio de estrategia de integración tecnológica solo es posible mediante el aprovechamiento de desarrollos externos, frente al modelo de propia generación que siempre ha utilizado la banca. Las principales innovaciones vienen determinadas por un conjunto muy amplio de tecnologías como por ejemplo los chatbots, la inteligencia artificial y machine learning, uso de la biometría, aplicaciones en la nube, big data, blockchain, API, uso de la voz, etc.

En este contexto de transformación, se puede situar el momento competitivo del sector financiero a través de dos factores clave, que son:

- Necesidad de revisión de los productos y servicios bancarios actuales. Se trata de una concepción anticuada y no pensada para el mundo digital, con unos costes insostenibles.

- Nuevo entorno de competencia, derivado de la entrada de nuevos players en la industria de las finanzas y los movimientos de la banca para adaptarse a ellos.

\section{Transformación de los servicios financieros actuales}

Actualmente, el día a día de una persona en el mundo occidental cuenta con una omnipresencia de productos financieros que le facilitan comodidad, seguridad y rendimientos para sus ahorros, y que han sido muy útiles hasta ahora en su vida corriente.

Siguiendo el esquema del ciclo financiero diario de una persona tipo bancarizada en Occidente, observamos que reside en una vivienda que adquirió gracias a un préstamo hipotecario de un banco, y se despierta con la alarma del teléfono móvil, cuyos pagos mensuales se llevan a cabo a través de su cuenta bancaria, en la que también se pagan los suministros de la luz, agua, gas, etc., lo que evita engorrosos desplazamientos a las compañías o envíos de dinero. A continuación, desayuna con alimentos que adquirió mediante el pago con una tarjeta de crédito. Al desplazarse al trabajo con su automóvil, de nuevo, el banco le ayudó con un préstamo vehículo a su adquisición. La nómina de su actividad laboral es ingresada por la empresa en su cuenta, y esto le aporta unas ventajas concretas en su banco. También al mediodía paga la comida en el restaurante mediante una tarjeta de crédito. Cuando llega a su casa, consulta la evolución de los ahorros para atender a futuros pagos y finalmente efectúa una transferencia para el pago de una actividad extraescolar de sus hijos. Estos son solo algunos ejemplos de la omnipresencia de 
las finanzas en nuestras vidas corrientes, y ha sido el modelo vigente en los últimos años, basado en productos bancarios contratados con presencia física y últimamente disfrazados de digitales con el uso de canales a distancia.

\section{Gráfico 1. Ciclo clásico de prestación de servicios financieros cotidianos}

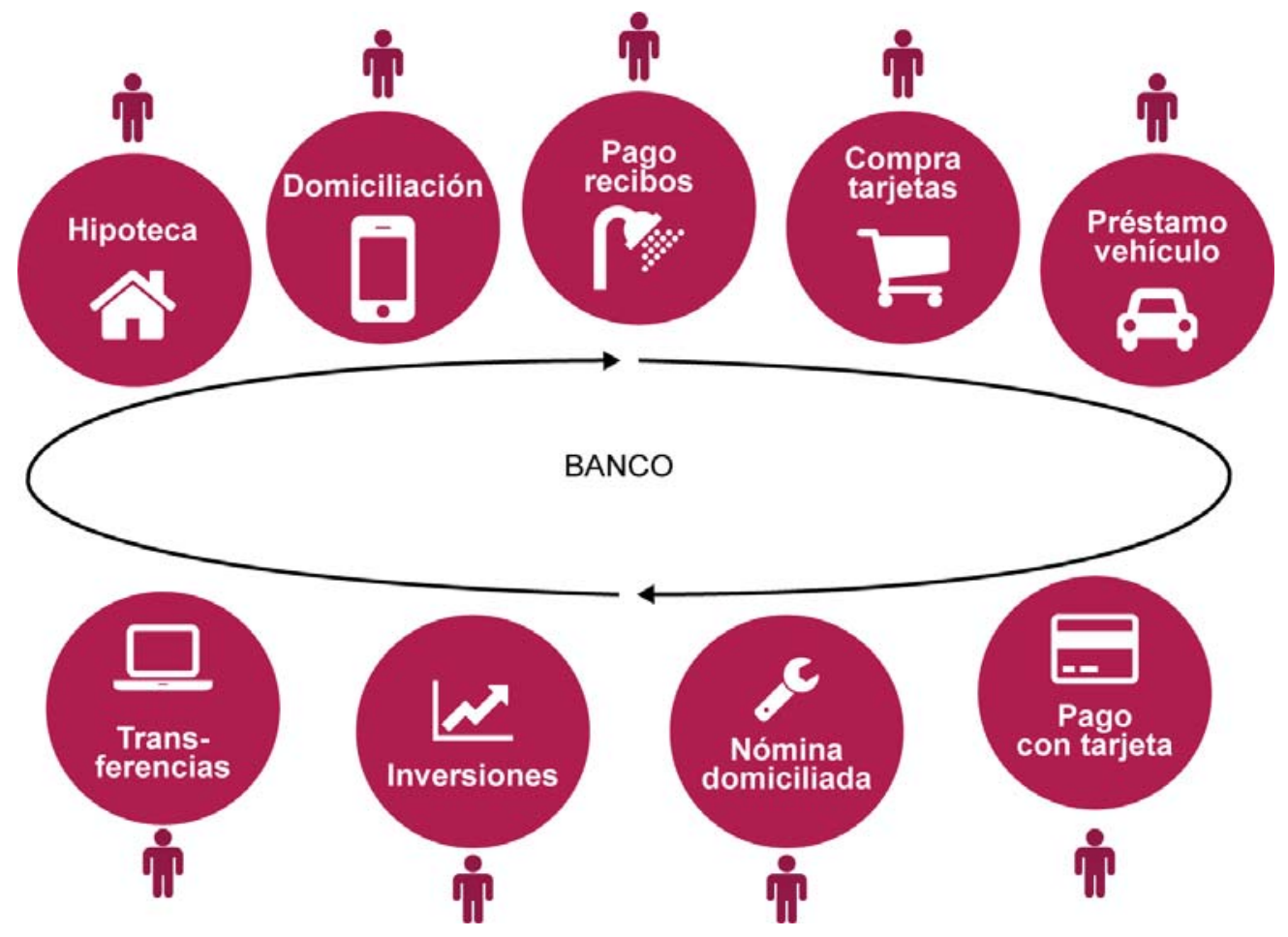

Fuente: Elaboración propia.

Dejando clara la gran utilidad de este modelo para los consumidores en el pasado, actualmente ya se identifican grandes déficits de estos servicios si se comparan con el estándar de empresas tecnológicas como Google, Facebook, Amazon, etc., que actúan como auténticos mitos tecnológicos y están marcando la experiencia de cliente mediante servicios de uso intuitivo, transparentes, ágiles y económicos para acceder a información y tomar decisiones inmediatas. Hoy día, se puede adquirir una prenda de ropa o un billete de avión con un solo clic. En cambio, para transacciones financieras como comprar acciones de una empresa del IBEX, se ha de superar un proceso muy complejo. Incluso para consultar un determinado recibo pagado en una cuenta bancaria, el proceso de búsqueda entre los apuntes no es nada fácil ni ágil. Es evidente la necesidad de redefinir el modelo de relación de la banca con sus clientes, basado en productos y aplicaciones individuales desconectadas, como si cada servicio fuera prestado a un cliente distinto, sin más valor que el propio pago (véase gráfico 1).

Estas carencias se hacen evidentes al analizar muchos de los actuales servicios, por ejemplo: 
- En relación con los suministros de luz, agua, gas, etc., las limitaciones del servicio bancario actual son muy evidentes. Se recibe un cargo en la cuenta para el pago de cada uno de los servicios. En cambio, para consultar el consumo es necesario ir al proveedor, identificarse de nuevo en la parte privada de clientes y consultar entonces los consumos, posibilidades de tarifas, etc. Si ya os encontráis identificados en la web del banco, ¿no puede este abrir una sesión con la compañía suministradora, en la que directamente sea posible consultar consumos, modificar tarifas, etc.? El servicio debería transformarse desde el actual pago simple sin valor añadido a un reinventado servicio de consulta y orientación de consumos. Nadie mejor que el banco conoce las características del hogar y los pagos que se hacen para poder ofrecer propuestas automatizadas.

- Si se considera el vehículo, de nuevo, el banco mantiene una información desagregada de todo lo relacionado con este aspecto de la movilidad: el préstamo vehículo, el impuesto de circulación, el seguro anual obligatorio (para un coche que los habitantes en las grandes ciudades utilizan de media menos de 50 días al año), los pagos de combustible, las reparaciones, las inspecciones y mantenimiento y las nuevas opciones de sustitución o renovación. Todos estos conceptos pasan por el banco de forma desagregada. ¿No sería posible disponer de un servicio global de estos puntos? Incluso antes de adquirir un nuevo automóvil, una información financiera del mismo.

- En las transferencias y los pagos a terceros, a pesar de las mejoras ya aplicadas, no se entienden algunos plazos de entrega o una comisión extra si se quiere acelerar el plazo de recepción. Por ello, algunos bancos se han dado cuenta de esta situación y ya ofrecen opciones de pago peer-to-peer (P2P) en colaboración con operadores especializados, ${ }^{7}$ mediante alternativas que son más baratas que la propia red bancaria Swift. Aunque las ventajas de las criptomonedas aún no son visibles completamente y la tecnología blockchain se encuentra en sus inicios, su aplicación puede marcar el cambio de tendencia en el negocio de transferencias y pagos a terceros.

Estos son solo algunos ejemplos que evidencian la necesidad de cambio hacia unos servicios que generen más valor para el usuario, y que solo pueden ser ofrecidos por entidades potentes como los bancos, con capacidad para actuar en entor-

7. JPMorgan Chase, a través de Finn, su oferta bancaria totalmente digital, dirigida a los usuarios de iOS en EE. UU., permite a los clientes abrir una cuenta, activar una tarjeta de débito de Chase que les da acceso a 29.000 cajeros automáticos, hacer pagos peerto-peer (P2P) a través de Zelle y utilizar una variedad de ofertas de gestión de finanzas personales dentro de la aplicación. 
nos seguros conectados con las grandes compañías suministradoras de servicios, administraciones, fuentes de información externas, etc., para ofrecer alternativas intuitivas, ágiles, transparentes y más económicas. Algunas entidades ya han iniciado este camino y ofrecen las primeras versiones de servicios integrados, principalmente en temas de gastos del hogar, si bien solo con una primera capa informativa. ${ }^{8}$ Otros sectores, como los operadores de telefonía, han sabido integrar en sus ofertas distintos servicios al cliente, como la telefonía móvil, telefonía fija, venta de aparatos, televisión por cable, fútbol, Netflix, etc. Las entidades financieras que no sean capaces de generar nuevo valor verán cómo sus productos son ofrecidos por nuevos agentes más ágiles, sin estructuras pesadas y que son capaces de ofrecer un servicio reinventado, más barato y eficiente (véase gráfico 2).

\section{Gráfico 2. Nuevo enfoque de los servicios financieros integrados}

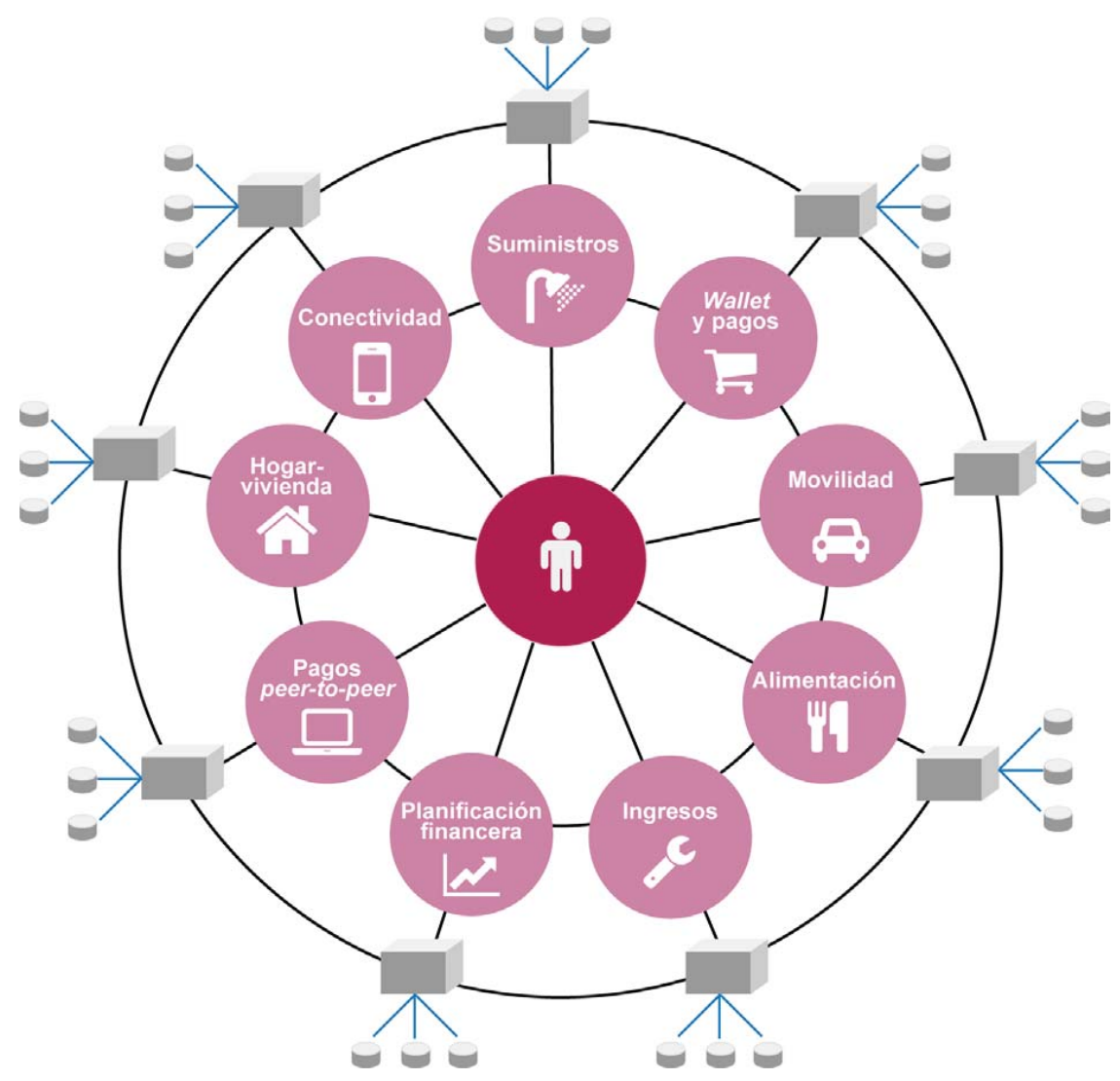

Fuente: Elaboración propia.

8. Los principales bancos del mundo han desarrollado primeras propuestas en este sentido. En España existen los ejemplos de Now de CaixaBank, Bconomy del BBVA o Money Plan del Banco Santander, que además permiten integrar información de otras entidades. 


\section{Los nuevos players}

Hay que distinguir claramente dos grandes grupos: en primer lugar, las fintech (finance + technology), y en segundo lugar, los gigantes tecnológicos conocidos como GAFA (Google, Apple, Facebook, Amazon), en Occidente, y BAT (Baidu, Alibaba y Tencent), con origen en China.

\subsection{Las fintech}

\subsubsection{Características de las fintech}

Las fintech son empresas innovadoras que ofrecen nuevas soluciones financieras con el apoyo de las nuevas tecnologías. Son auténticas especialistas en áreas concretas de los servicios financieros, y parten de un formato start-up. Se pueden destacar las siguientes características:

\section{Gráfico 3. Características de las fintech}

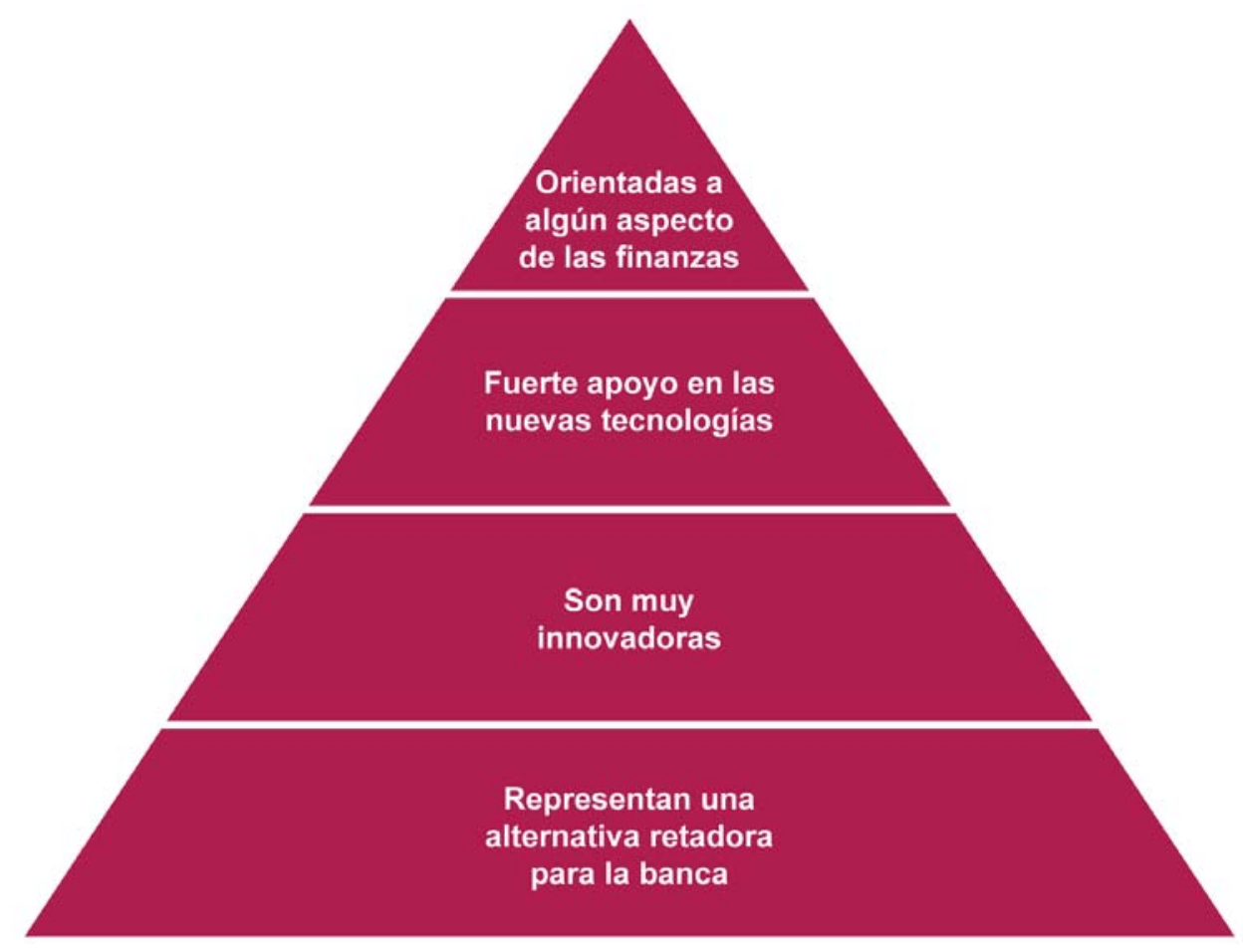

Fuente: Elaboración propia.

- Su propuesta está centrada en algún aspecto concreto de las finanzas. Esta simplicidad les otorga una oferta muy vertical de la actividad financiera, que contrasta con la oferta multiproducto de los bancos.

- Mediante el uso intensivo de nuevas tecnologías, las fintech ofrecen soluciones a problemas financieros de los clientes o a necesidades mal atendidas. Utilizan 
innovadoras plataformas tecnológicas (principalmente a través de dispositivos móviles), con aplicaciones intuitivas y amigables.

- Las empresas fintech proceden de la cultura de la innovación y de startup, creadas desde cero, con una filosofía de romper con los anteriores formatos. Conviven y se desarrollan en un contexto de redes sociales, con una cultura peer-to-peer más que de sumisión a una entidad poderosa como la que representan los bancos.

- Se plantean, en su inicio, como una alternativa retadora respecto a la banca tradicional. Su oferta es combativa respecto al producto bancario, y uno de sus argumentos principales es presentarse como una opción nueva, más eficiente y transparente que los productos ofrecidos por la banca.

\subsection{2. Ámbitos de actividad de las fintech}

Los ámbitos de actuación de las fintech son diversos y cubren mediante nuevos enfoques casi todas las líneas clásicas de los servicios financieros, con innovadoras propuestas más económicas, transparentes e intuitivas digitalmente. De acuerdo con los sectores de actividad a los que están orientadas, se pueden establecer diferentes grupos operativos, también denominados verticales. No hay un criterio único para esta agrupación, ${ }^{9}$ y en este artículo consideraremos diez agregaciones:

\section{- Medios de pago}

Empresas que ofrecen soluciones sobre medios de pago electrónicos, evitando el empleo de dinero físico y de manera independiente respecto a los bancos.

\section{- Financiación de particulares y empresas}

Se incluyen en este vertical las fintech dedicadas a organizar un marketplace en el que participan solicitantes de financiación e inversores que prestan recursos económicos. Son las denominadas crowdlending (préstamos) y crowdequity (capital), y pueden ser operaciones entre particulares (P2P) o bien entre particulares y empresas (P2B).

\section{- Inversiones - roboadvisor}

El sector de la inversión y el asesoramiento personal, típico de la banca, ha sufrido una disrupción con la llegada de los servicios de inversión automatizados,

9. La Asociación Española de Fintech e Insurtech establece 13 agrupaciones. Finnovating utiliza 14 verticales y 29 subverticales. En el Reino Unido, se distinguen 8 grandes verticales y 35 subverticales. 
conocidos como plataformas de inversión automatizadas. Presentan menores costes de gestión, un análisis de las opciones de inversión carente de subjetividad y una fuerte capacidad para el rebalanceo de la cartera.

\section{- Infraestructura y seguridad}

Se trata de startups que han desarrollado innovaciones en temas de seguridad, aprovechamiento de la tecnología y gestión de la identidad digital. Aportan gran utilidad en procesos de alta en línea y de compliance.

\section{- Big data}

Cuando una empresa emplea un enfoque data-driven ('impulsado por datos'), toma decisiones estratégicas basadas en análisis de datos e interpretación. La orientación de la actividad general de un banco le puede impedir profundizar en aspectos concretos que se identifican para establecer patrones de comportamiento, algoritmos predictivos y otras utilidades. En muchos casos, incorporan también soluciones de inteligencia artificial.

\section{- Criptomonedas y blockchain}

El bitcoin, las criptomonedas y, principalmente, la tecnología blockchain han abierto un nuevo campo en las finanzas, con múltiples opciones y aplicaciones, y el ámbito de las fintech es en el que más se han impulsado en un inicio. Este es el contenido al que se están dedicando principalmente las últimas creaciones de empresas, y el que se considera que va a tener más influencia en los próximos años.

\section{- Finanzas personales}

En este vertical, aparecen fintech que ofrecen una gestión integrada de las finanzas personales, agrupan la información que pueden aportar los diferentes bancos que puede tener un cliente y llevan a cabo propuestas y recomendaciones orientadas a facilitar la operativa financiera. La nueva legislación, vigente desde enero del 2018, sobre la Directiva PSD2 (Payment Services Directive 2) permite la apertura por parte de los bancos de sus servicios de pagos a terceras empresas y a los proveedores de servicios de información externos.

\section{- Servicios de transacciones y divisas}

Engloba a empresas que ofrecen opciones transaccionales como transferencias, cambios de divisas, etc., generalmente en mejores condiciones que la banca. 


\section{- Insurtech}

Se incluyen en este grupo empresas que, aplicando la tecnología, son capaces de ofrecer coberturas de seguros mediante nuevos y flexibles enfoques.

\section{- Neobanks y challenger banks}

En esta actividad se incluyen entidades que ofrecen productos financieros, no siempre con una licencia bancaria, pero con la característica común de trabajar solo con canal smartphone, apoyo de redes sociales y enfoque data-driven.

Después de unos años en funcionamiento, la actuación de las fintech ha evolucionado y se pueden destacar las siguientes tendencias concretas en cuanto al contenido de su actividad:

- Procesos de fusión para alcanzar masa crítica en actividades como crowdlending ${ }^{10}$ por problemas de escalabilidad.

- Ampliación de la actividad para cubrir un mayor ámbito del mercado, como el caso de los roboadvisor con la incorporación de los quantadvisors, los cuales, manteniendo el uso de algoritmos, actúan mediante estrategias de gestión activa y búsqueda de retornos positivos, estables e independientes de los mercados financieros, frente a la pasiva o indexada de los roboadvisors.

- Actuaciones full-stack entre fintech de diferentes verticales, para orientar la tecnología a solucionar problemas concretos como, por ejemplo, la utilización de la inteligencia artificial en los pagos con el objeto de control del fraude.

- Escaso interés de los bancos en los ámbitos de criptomonedas. Dejan la actividad de exchange, ICO, etc. a operadores independientes.

- Ampliación de los casos de uso conjunto de fintech con bancos en la tecnología blockchain.

10. En España, el volumen financiado por el conjunto de estas empresas ascendía en el 2017, según la Asociación Española de Crowdlending (10 empresas), a 100 millones de euros de préstamos concedidos, que es una cifra muy baja frente a los 42.000 millones de euros de crédito al consumo concedidos por la banca. Este volumen contrasta con los niveles conseguidos en otros países como EE. UU., con el liderazgo de LendingClub (más de 2,4 millones de clientes y 35 billones de dólares concedidos en préstamos) o Prosper (13 billones de dólares concedidos y 0,8 millones de clientes). Una visión distinta es la aportada por Marc Montemar y Helena Benito (nota técnica IEF número 35), que indican que el bajo nivel de España del crowdfunding se debe a que aún no se ha desarrollado suficientemente (volumen per cápita que mueve el crowdfunding en España: 2,82 €; respecto a 6,64 € en Francia o hasta los 85,44 € en el Reino Unido). 
- Desarrollos en los ámbitos de regtech e insurtech.

- Orientación a la colaboración de los bancos con las fintech, principalmente en los verticales de infraestructura, seguridad y big data.

- Empezar a considerar la incorporación de préstamos hipotecarios en línea (no permitidos en España mediante el crowdlending en las actuales plataformas participativas).

\subsubsection{Impacto de las fintech en la actividad bancaria}

Si bien es ingenuo pensar que las fintech y los nuevos agentes que están apareciendo en el mundo financiero pueden desbancar a la banca a corto y medio plazo, también es cierto que los bancos que no los tengan en cuenta pueden quedarse al margen de las nuevas ideas e innovaciones que están aportando muchas de estas dinámicas empresas.

El volumen operativo actual de las fintech es pequeño con relación a las cifras del negocio financiero. No obstante, su influencia y crecimiento son muy notables. En un ámbito mundial, se estima que existen unas 15.000 fintech, con EE. UU. y Reino Unido liderando el sector, mientras que en España el ecosistema cuenta con más de 300 empresas y 5.000 trabajadores y se encuentra dentro de los 10 primeros países del mundo por número de fintech. ${ }^{11}$ Si bien en un inicio su planteamiento frente a la banca era el de ser alternativa, esta opción se ha ido modificando, y actualmente su objetivo es en muchos casos el de colaboración e incluso complementariedad.

El nivel de colaboración o competencia de las fintech se puede analizar desde diferentes puntos de vista, si bien los resultados son bastante homogéneos:

- En primer lugar, hay que considerar el modelo de negocio de la fintech, que puede ser B2B (negocio dirigido a empresas), el cual busca la colaboración con los bancos, o bien B2C (negocio orientado a particulares), que se orienta al consumidor final y su oferta es una alternativa al sector bancario.

- La percepción sobre la complementariedad, colaboración o competencia, según la vocación de la propia fintech.

- Distinguir entre las fintech que colaboran realmente, las que están en negociaciones y las que no colaboran ni lo tienen previsto.

A partir del modelo de negocio en España, 5 de los 10 verticales considerados (véase tabla 1) plantean un servicio dirigido al consumidor directamente con una

11. Finnovating (2018). Observatorio de innovación y tendencias fintech. 
actividad en competencia directa con la banca. No obstante, hay que tener en cuenta que, a diferencia de España, en otros países como EE. UU. o el Reino Unido, las actividades como roboadvisor y crowdfunding también son participadas intensamente por la banca. Por otra parte, si se considera el total de las empresas, ${ }^{12}$ el $52 \%$ de las fintech españolas tienen un modelo B2B, mientras que un $34 \%$ tienen un modelo B2C en exclusiva.

Tabla 1. Relación de verticales de fintech y modelo de negocio

\begin{tabular}{|c|c|c|}
\hline Vertical & Modelo de negocio & Actitud con la banca \\
\hline Medios de pago & B2C & $\begin{array}{l}\text { Competencia y } \\
\text { colaboración }\end{array}$ \\
\hline $\begin{array}{l}\text { Financiación de } \\
\text { particulares y empresas }\end{array}$ & B2C & Competencia \\
\hline Roboadvisor & B2C & Competencia \\
\hline Infraestructura y seguridad & B2B & Colaboración \\
\hline Big data & $\mathrm{B} 2 \mathrm{~B}$ & Colaboración \\
\hline $\begin{array}{l}\text { Criptomonedas y } \\
\text { blockchain }\end{array}$ & $\mathrm{B} 2 \mathrm{~B}$ & Complementariedad \\
\hline Finanzas personales & $\mathrm{B} 2 \mathrm{~B}$ & Complementariedad \\
\hline $\begin{array}{l}\text { Servicios transaccionales } \\
\text { y divisas }\end{array}$ & $\mathrm{B} 2 \mathrm{C}$ & Competencia \\
\hline Insurtech & $\mathrm{B} 2 \mathrm{C}$ & Complementariedad \\
\hline $\begin{array}{l}\text { Neobanks y challenge } \\
\text { banks }\end{array}$ & $\mathrm{B} 2 \mathrm{C}$ & Competencia \\
\hline
\end{tabular}

Fuente: Elaboración propia.

Atendiendo al número total de empresas fintech que tienen vocación de colaboración, diferentes estudios ${ }^{13}$ apuntan que la proporción que se orienta a la asociación

12. Funcas-KPGM (2018). Observatorio de divulgación financiera.

13. Según el Observatorio de innovación y tendencias fintech del 2018 de Finnovating, se estiman en un $75 \%$ las fintech que colaboran con la banca. De la misma manera, en el estudio Comparativa de la oferta de la banca vs. fintech de Funcas y KPMG del 2018 se 
con los bancos (que incluye complementariedad o colaboración) es de un 75$80 \%$, mientras que la competencia directa queda reducida a un 20-25 \%.

\subsection{La amenaza de los gigantes tecnológicos}

A diferencia de las pequeñas fintech, las grandes compañías tecnológicas sí representan una auténtica amenaza para las entidades financieras, tanto por su capacidad de generar innovadores servicios muy intuitivos basados en nuevas tecnologías, como por su poder de alcance, con millones de clientes cautivos de sus productos. Su poder económico también es muy grande, y en pocos años se han situado entre las mayores empresas del mundo por capitalización. No obstante, los bancos no están indefensos ante estas compañías, ya que sus puntos fuertes de seguridad y regulación son precisamente los puntos débiles de algunas de estas bigtech, y los riesgos se han puesto de manifiesto recientemente, con el ejemplo de los problemas de seguridad de datos que ha tenido Facebook. ${ }^{14}$

Además de los citados GAFA (Google, Amazon, Facebook y Apple) y BAT (Baidu, Alibaba y Tencent), también se podría incluir a Samsung y Microsoft como bigtech que ya tienen servicios financieros operativos. Muchas de estas grandes compañías tienen en común el hecho de que han lanzado sus propios sistemas de pago con tecnología NFC (Near Field Comunication), con plataformas exclusivas que permiten pagos con móvil mediante contactless, y a las cuales a los bancos no les queda más remedio que sumarse si quieren que sus clientes con un dispositivo de estas marcas puedan hacer pagos (como es el caso de Apple Pay, Samsung Pay o Google Pay, que tiene una apuesta clara no solo para el pago mediante móvil, sino también para comercio electrónico, con un gran potencial de desbancar a la banca en esta área). Amazon dispone de su propio sistema para efectuar pagos, préstamos, seguros, cheques, comercio electrónico, etc. Es decir, Amazon está ofreciendo servicios financieros desde todos los ángulos, sin ser un banco convencional (véase gráfico 4).

calcula que de las 300 fintech nacionales, actualmente el $48 \%$ de las compañías son complementarias a los bancos, el $32 \%$ son colaborativas y el $20 \%$, competidoras.

14. La consultora Cambridge Analytics se hizo en la última campaña de las elecciones en Estados Unidos con datos privados de usuarios de Facebook, lo que provocó un gran impacto negativo y la falta de confianza en esta compañía. Se han reconocido errores que provocaron la fuga de datos de 50 millones de usuarios. 


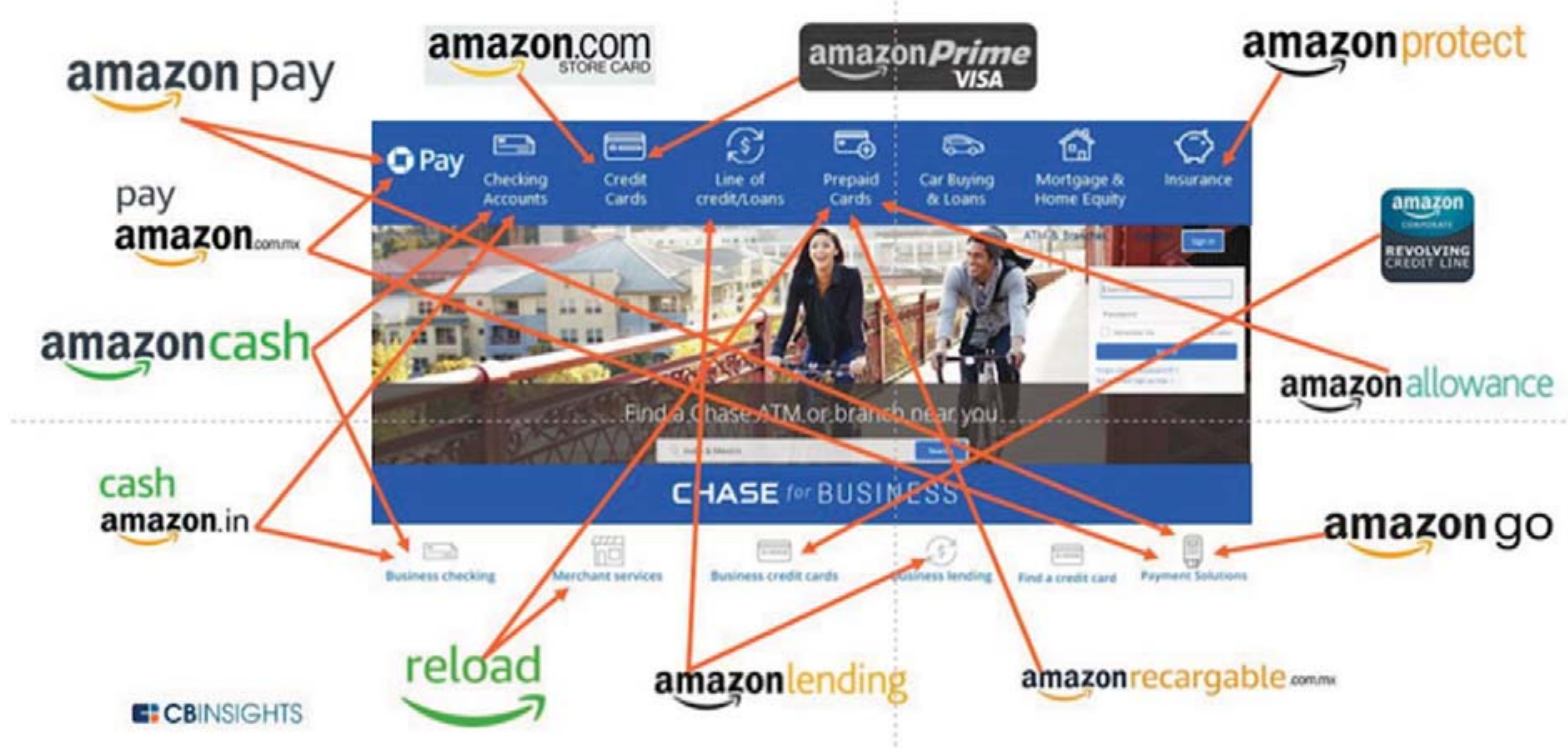

Fuente: CB Insights.

Facebook cuenta con WhatsApp, que ya tiene un sistema de pagos para transferir dinero entre los contactos de la aplicación. Con este sistema de pagos de la aplicación de mensajería instantánea, se puede enviar y recibir dinero de forma análoga a un mensaje de texto, una nota de voz o cualquier otro formato de contenido a través de WhatsApp. No obstante, hasta el momento, este servicio solo es exclusivo para la India, donde ya dispone de más de un millón de usuarios.

Sin embargo, las actuaciones de las bigtech se han centrado en el ámbito de los pagos, que han sido una de las áreas clásicas de negocio de la banca. Hasta ahora el impacto ha incidido en la reducción de las tasas bancarias, pero no en la suplantación de estas entidades, ya que al final hay que recurrir a una cuenta bancaria que actúa de depositario de los recursos económicos de los usuarios, pero la amenaza es evidente, y el papel de los bancos puede quedar muy relegado.

Otro aspecto en el que las entidades financieras pueden ser desplazadas es el de los micropréstamos, el cual, hasta ahora, ha tenido una utilización para segmentos marginales, pero que puede llegar a ser muy masivo, con una consiguiente reducción de los costes financieros para los clientes, pero una clara pérdida de negocio para los bancos. Actualmente, el crédito al consumo en la banca incluso supera en volumen al del préstamo hipotecario. No obstante, ¿qué sucedería si la financiación se desvincula del banco y se incorpora a los propios productos de consumo mediante multitud de micropréstamos, tal y como ya se hace para adquirir un móvil?

En este grupo de grandes operadores que suponen una amenaza para la banca, hay que considerar también a los operadores de telefonía como Vodafone, 
Orange o Movistar en España. Orange ya estaba presente en la financiación a plazos de teléfonos en pólizas de seguros para teléfonos móviles inteligentes y con Orange Cash para pagos, y recientemente ha creado un banco que ya es operativo en Francia y espera iniciar su actividad en España en el 2019. ${ }^{15}$

\section{La respuesta de la banca}

Después de la primera generación de fintech que mantenían posiciones muy radicales de disrupción total con la banca tradicional, la actitud ha cambiado y el número de las mismas que está colaborando con los bancos aumenta, porque estos también creen necesario el cambio. Se estima ${ }^{16}$ que el $90 \%$ de los bancos disponen de diferentes programas de colaboración con empresas fintech para promover la innovación en la propia entidad. Esta proporción se eleva al 100 \% en el caso de los grandes bancos. Los bancos más activos en un ámbito mundial ${ }^{17}$ desde el punto de vista de transformación digital, incluso, disponen de colaboraciones en todos los verticales posibles. El ámbito que genera más acuerdos de colaboración de las entidades bancarias con las fintech es el de «pagos a terceros», para explorar nuevos formatos y que demuestra la voluntad de defender esta área de negocio, aunque sea a costa de sacrificar productos actuales.

La colaboración entre las fintech y los bancos puede aportar valor complementario a los dos tipos de compañías. Por un lado, las fintech, con su orientación a la tecnología y capacidad de explorar caminos nuevos, pueden generar nuevas ideas. En cambio, no disponen de licencia bancaria para determinadas operaciones, ni de capacidad para escalar un servicio hasta alcanzar la masa crítica necesaria. Juntos, fintech y bancos pueden crear un nuevo ecosistema que les permita cubrir mejor las necesidades de sus clientes. También se observa la inversión de los bancos en neobancos o incluso en desarrollos propios independientes de la entidad tradicional, con el objetivo de crear sus propios «rivales digitales puros», para disponer de

15. Expansión (28 de abril del 2018). «La banca digital de Orange revoluciona el sector en Francia. De noviembre del 2017 a marzo del 2018, el nuevo servicio ya había captado 100.000 clientes en Francia. En España se prevé su inicio en el 2019 y la operadora está integrando en su equipo a directivos procedentes de la banca española».

16. Según el último informe de EBA, las acciones de colaboración alcanzan a cerca del 90 \% de las entidades financieras.

17. JP Morgan Chase \& Co. y Citibank Goldman Sachs disponen de acuerdos con fintech en todos los verticales. A continuación, se encuentran Bank Of America, Morgan Stanley y Wells Fargo (top ten de los bancos de EE. UU., según Bankingtech). 
marcas combativas que puedan competir con iniciativas similares de otros grupos o las propias bigtech y disponer de entidades digitales expertas y nuevas que quizá en el futuro puedan incluso suplir la matriz, ${ }^{18}$ como sucedió en otros sectores (por ejemplo, la marca digital Movistar suplantó a su generadora Telefónica).

Hay diferentes tipos de acuerdos posibles con las fintech, entre los cuales podemos destacar compras directas, creación de incubadoras y aceleradoras, asociaciones, prestación de servicios y acuerdos de asociación (véase el gráfico 5).

Gráfico 5. Tipos de acuerdos de los bancos con las fintech

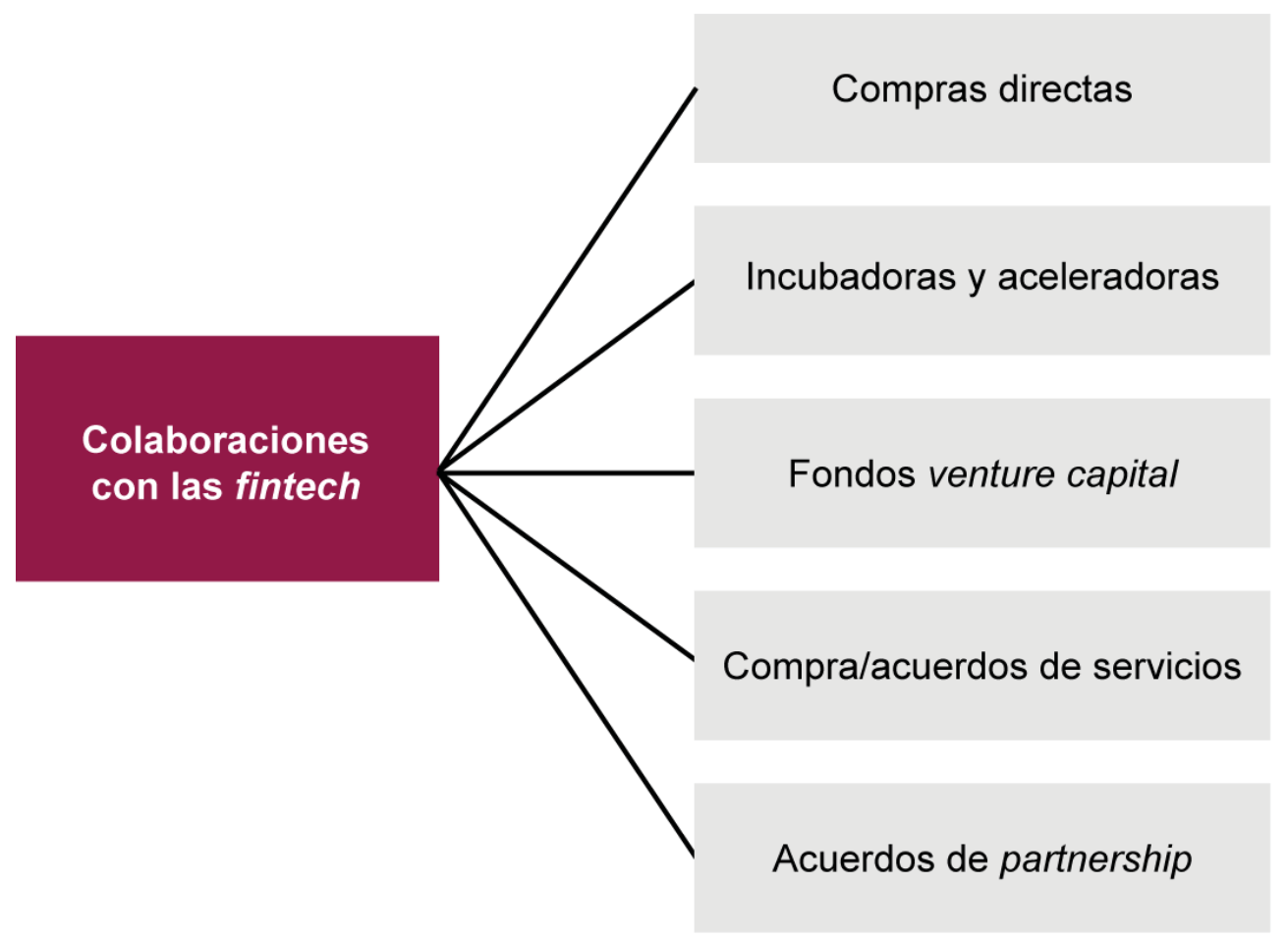

Fuente: Elaboración propia.

Evidentemente, las entidades bancarias no pueden estar en todas las fintech ni en todos los ámbitos. Para ello, la estrategia de las colaboraciones está teniendo en cuenta dos variables determinantes: por un lado, la rivalidad que significa para el negocio y, por otro, la capacidad de impactar en volumen.

De acuerdo con esta dualidad, se puede construir la matriz de alianzas que ayuda a entender la estrategia que hay que seguir. Consiste en incorporar en el core del banco a aquellas startups que se prevea con fuerte impacto de rivalidad y capacidad de conseguir volumen. Para las fintech con poca capacidad de cre-

18. En este grupo, se enmarcan las actuaciones de Santander con Open Bank o de BBVA con Simple o Atom Bank. 
cimiento en negocio, pero que supongan una fuerte amenaza por la rivalidad, la propuesta es mantener su desarrollo a través de los programas de aceleradoras e incubadoras del banco, hasta ver su potencial. Finalmente, se propone una política de alianzas con aquellas que tengan una capacidad de alcanzar volumen, pero que no sean competencia directa.

\section{Gráfico 6. Matriz estratégica de alianzas de la banca con las fintech}

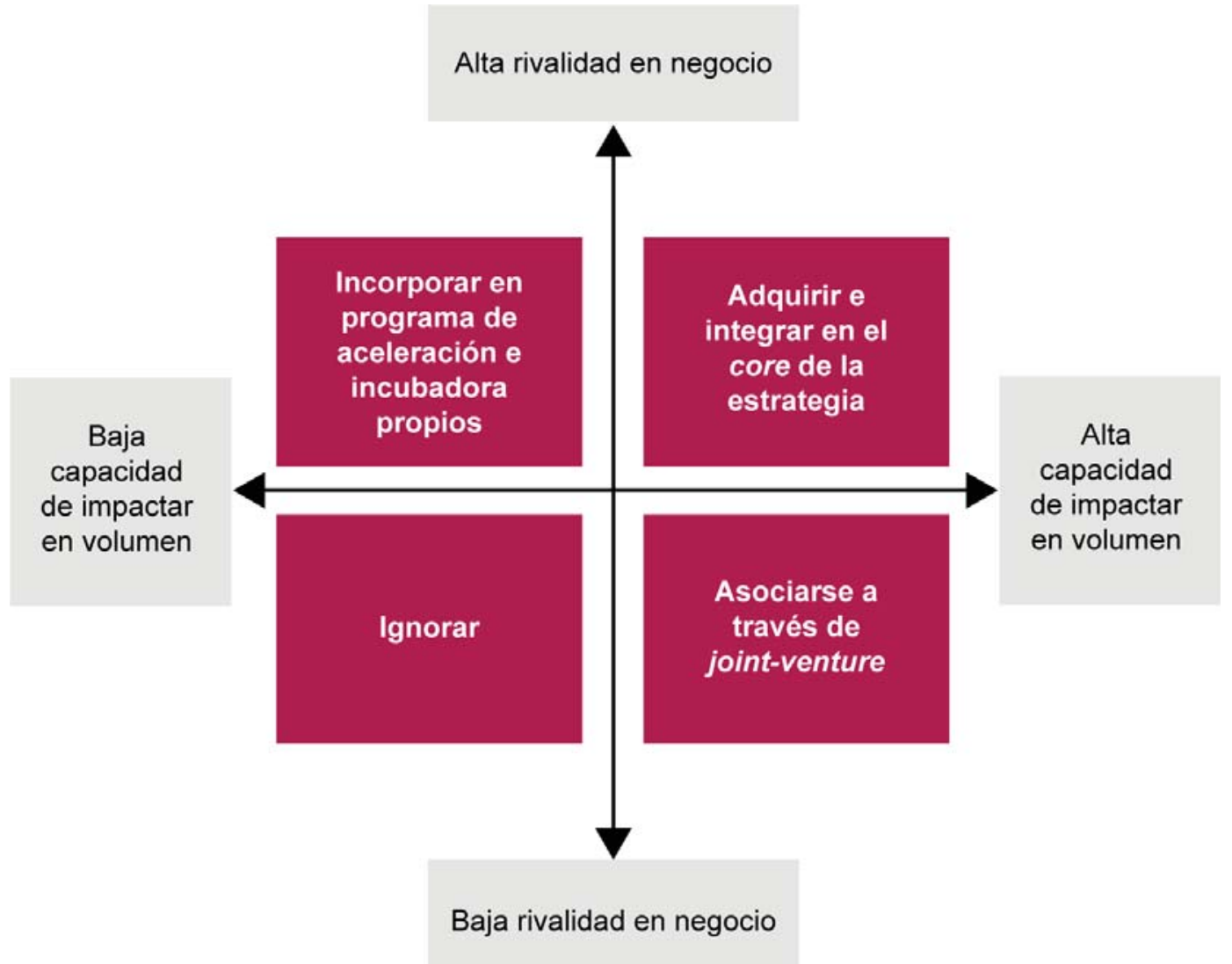

Fuente: Elaboración propia.

\section{Conclusiones}

Los motores de cambio en el sector bancario son cada vez más intensos. Los productos bancarios requieren una reinvención para ajustarse a los cambios en la demanda de los nuevos consumidores digitales, y las propias entidades financieras se encuentran en niveles no sostenibles de rentabilidad, con estructuras ineficientes. Todo esto lleva a una fuerte velocidad de cambio digital, junto con la aparición de un conjunto amplio de innovaciones tecnológicas como inteligencia artificial y machine learning, blockchain, biometría, cloud computing, API abiertas, big data, etc., que obligan a una apertura en la innovación frente al modelo de desarrollo interno que tenía la banca en el pasado. Además, la aparición de las fintech y la 
presión de las bigtech constituyen una amenaza, ya que estas empresas son capaces de ofrecer servicios financieros alternativos más intuitivos, agiles, transparentes y más baratos que los productos bancarios.

La única opción para la banca es la transformación mediante la reinvención de sus servicios, o bien los ofrecerán los nuevos players que ya hacen propuestas, con notable éxito, en áreas de negocio colindantes como los pagos de compras, el envío de dinero, la microfinanciación, etc. En este contexto, los bancos aún disponen de distintos puntos de anclaje en su favor. El primero de ellos es su fortaleza y la disposición de información financiera completa de sus clientes (solo tienen que integrarla y convertirla en valor añadido, frente al actual modelo de cobro-pago desagregado). En segundo lugar, disponen de un reconocimiento en la gestión de protección de datos de sus clientes, que es un valor muy alejado de la compartición y poca seguridad atribuibles a algunas tecnológicas. Finalmente, la misma regulación que les ocasiona grandes costes, al perseguir la protección del consumidor, puede ser también su gran aliada, ante la incertidumbre de propuestas no validadas oficialmente.

Así como la amenaza de las bigtech es cada vez mayor, con propuestas financieras propias que les ayudan a vender más (Amazon, Apple, Google, etc.) y a prescindir de los bancos, el panorama de las fintech ha cambiado respecto a su origen. Paradójicamente, estas entidades, que eran retadoras y cuyas propuestas se presentaban como alternativas a la banca en su origen, en su mayor parte se han convertido en aliadas de la banca, al encontrarse con problemas de escalabilidad, y por ello se orientan a la colaboración o complementariedad con el sector financiero, y están ayudando de manera clara a que el cambio en los bancos sea más rápido y más transformacional que disruptivo. Solo entre el 20-25 \% de las fintech plantean una rivalidad que en un ámbito de negocio es irrelevante, y con problemas de crecimiento.

La gestión del cambio de las finanzas se está desarrollando por parte de los bancos mediante múltiples estrategias, con inversiones propias, adquisiciones, acuerdos, etc., para integrar la tecnología y transformar los servicios. El análisis correcto de cada área de innovación para identificar el impacto en volumen de negocio, así como la rivalidad para desplazar actuales servicios, serán las claves del éxito. El resultado apunta a una conversión de los bancos en plataformas diversificadas con servicios globales de valor añadido y con una experiencia mobile-first y data-driven, e impulsados por acuerdos con empresas externas fintech para no frenar la disrupción, pero con una integración controlada. 


\section{Referencias bibliográficas}

ÁLVAREZ, C. (2018). La biometría y los pagos NFC, las tecnologías favoritas de la banca europea. BBBAworld / Fintech EBA.

BI INTELLIGENCE RESEARCH TEAM (2018). 35 big tech predictions for 2018. BI Intelligence.

CHANDRASHEKAR, A.; KUMAR, A.; SAXENA, A. (2018). Top 10 Trends in Retail Banking 2018. CAPGEMINI.

COURBE, J. (2016). PWC, Financial Services Technology 2020 and Beyond: Embracing disruption. PWC.

EFMA, C. (2018). World Retail Banking Report 2017.

EUROPEAN BANKING AUTHORITY (EBA) (2018). Report on the impact of fintech on incumbent credit institutions'business models.

EUROPEAN BANKING AUTHORITY (EBA) (2018). Report on the prudential risc and opportunities arising for institutions from fintech.

FINNOVATING DISRUPTING CORPORATIONS (2018). Observatorio de innovación y tendencias fintech 2018.

IGUAL, D. (2016). Lo quelatecnología haceporlas finanzas. Barcelona: Profit Editorial. INDRA (2017). La nueva banca, una plataforma al servicio de tu bienestar financiero. LACROIX, C. (2017). How Fintechs are Forcing Banks To Engage Into Digital Transformation. Cap Gemini.

MALTZAHN L. (2017). Future-Ready Bank. Creating the conditions for Banking succes in the digital age. Accenture.

MCWATERS, R.; BRUNO, G. (2017). «Beyond Fintech: A Pragmatic Assessment Of Disruptive Potential In Financial service». Part of the future of financial services series / Prepared in collaboration with Deloitte. World Economic Forum.

MONTEMAR, M.; BENITO, H. (2018). «Evolución del Equity Crowdfunding en España, 2011-2017». Observatorio de divulgación financiera. Institut d'Estudis Financers (IEF).

NGUYEN, M. H. (2017). «How chatbots and artificial intelligence will save banks and the finance industry billions». Business Insider. BI Intelligence.

OBSERVATORIO DE DIVULGACIÓN FINANCIERA (2018). Comparativa de la oferta de la banca vs. Fintech. Funcas y KPMG. 


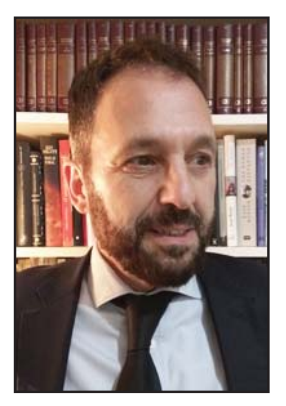

\section{David Igual Molina david.igual@gmail.com Profesor de la BSM-UPF. Profesor colaborador de la UOC}

Licenciado en Ciencias Económicas y Empresariales por la Universidad de Barcelona y máster en Dirección y gestión de marketing por ESADE. Su trayectoria profesional se ha desarrollado en entidades financieras, con responsabilidades en diferentes áreas de negocio y productos. Profesor en diferentes programas nacionales e internacionales, y ponente en eventos sobre productos bancarios y cambio tecnológico en las finanzas. Es socio fundador y director de Operaciones de Pensium. Ha publicado diferentes libros sobre productos financieros, así como el primero que se publicó en España sobre fintech.

Los textos publicados en esta revista están sujetas -salvo que se insdique el contrario- a una licencia de Reconocimiento 3.0 España de Creative Commons. Podéis copiarlos, distribuirlos, comunicarlos públicamente y hacer obras derivadas siempre que reconozcáis los créditos de las obras (autoría, nombre de la revista, institución editora) de la manera especificada por los autores o por la revista. La licencia completa se puede consultar en http://creativecommons.org/licenses/by/3.0/es/deed.ca.

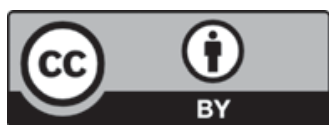

